OPEN ACCESS

Edited by:

Jung Ryeol Lee,

Seoul National University College of

Medicine, South Korea

Reviewed by:

Young Sik Choi,

Yonsei University, South Korea

Seido Takae

St. Marianna University School of

Medicine, Japan

Jongkil Joo,

Pusan National University Hospital,

South Korea

*Correspondence:

Dong-Yun Lee

dongyun0406.lee@samsung.com

Specialty section:

This article was submitted to

Reproduction,

a section of the journal

Frontiers in Endocrinology

Received: 13 December 2018

Accepted: 25 March 2019

Published: 11 April 2019

Citation:

Son K-A, Lee D-Y and Choi D (2019) Association of BRCA Mutations and Anti-müllerian Hormone Level in Young Breast Cancer Patients.

Front. Endocrinol. 10:235

doi: 10.3389/fendo.2019.00235

\section{Association of BRCA Mutations and Anti-müllerian Hormone Level in Young Breast Cancer Patients}

\author{
Kyung-A Son, Dong-Yun Lee* and DooSeok Choi \\ Department of Obstetrics and Gynecology, Samsung Medical Center, Sungkyunkwan University School of Medicine, Seoul, \\ South Korea
}

Background: Several preclinical and clinical studies have suggested that BRCA-mutation carriers may have decreased ovarian reserve. However, data in this area are limited and inconsistent, especially in young breast cancer patients.

Objective: This study evaluated the association between BRCA mutation status and serum anti-Müllerian hormone (AMH) level in young, reproductive-aged patients with breast cancer.

Materials and Methods: Patients $\leq 40$ years of age with breast cancer and who had known BRCA status and baseline serum AMH level at Samsung Medical Center, Seoul, Korea, were considered for inclusion. A total of 52 BRCA mutation carriers (27 BRCA1 and 25 BRCA2) and 264 non-carriers were selected for analyses. The serum level of $\mathrm{AMH}$ was compared according to presence of a BRCA mutation, and linear and logistic regression analyses were performed to evaluate the association between $B R C A$ mutation and serum $\mathrm{AMH}$ level.

Results: No difference was found in clinical characteristics between BRCA-mutation carriers and non-carriers. Subjects with any BRCA mutation had a significantly lower median $\mathrm{AMH}$ than those without a mutation $(2.60 \mathrm{vs} .3 .85 \mathrm{ng} / \mathrm{mL}, 32 \%$ reduction, $P=0.004)$. Linear regression analysis showed a significant negative association between $B R C A$ mutation and $\mathrm{AMH}$ level. In addition, logistic regression demonstrated non-significantly increased odds of mutation carriers having $\mathrm{AMH}<1.2 \mathrm{ng} / \mathrm{mL}$. However, no difference was found between BRCA1/2 mutations.

Conclusions: Breast cancer patients with BRCA mutation have significantly lower serum $\mathrm{AMH}$ level. Fertility preservation should be considered more aggressively in young breast cancer patients with BRCA mutation.

Keywords: breast cancer, anti-Müllerian hormone, BRCA1, BRCA2, ovarian reserve

\section{INTRODUCTION}

$B R C A$ mutations are associated with high risk of breast and ovarian cancer in reproductive-aged women $(1,2)$. The lifetime risks of breast and ovarian cancer are 65 and 39\%, respectively, in BRCA1 mutation carriers and 45 and $11 \%$ in BRCA2 mutation carriers (3).

In addition to cancer risk, it has been suggested that BRCA mutation may be related to decreased ovarian reserve, due to BRCA's function in repairing double-strand 
DNA breaks (4). Several studies have demonstrated significantly decreased serum anti-Müllerian hormone (AMH) level, a biomarker representing ovarian reserves (5), in BRCA mutation carriers (6-9). Moreover, in breast cancer patients who underwent ovarian stimulation for fertility-preservation, there was a higher rate of poor ovarian response (POR) in BRCAmutation carriers compared to non-carriers $(10,11)$. However, some studies have found no difference in serum AMH level according to BRCA mutation status (12-15). Therefore, the association between BRCA mutation status and decreased ovarian reserve is not conclusive. In addition, only a few studies have shown a significant association between $B R C A$ mutation and decreased ovarian reserve in young breast cancer patients $(16,17)$.

Considering that it is currently recommended for $B R C A$ mutation carriers to complete childbearing by age 40 and to undergo a risk-reducing salpingo-oophorectomy, and that breast cancer patients with $B R C A$ mutation are at increased risk of infertility as a result of anticancer treatment (18), issues of fertility preservation should be a priority for young patients.

Therefore, this study aimed to clarify the relationship between $B R C A$ mutation and the level of ovarian reserve by comparing serum AMH level between BRCA-mutation carriers and noncarriers in breast cancer patients.

\section{MATERIALS AND METHODS}

This retrospective study included all premenopausal patients $\leq 40$ years of age who were diagnosed with breast cancer and had a known baseline status regarding $B R C A$ mutation and serum AMH level at Samsung Medical Center, Seoul, Korea, from December 2011 to May 2018. We excluded patients who (1) no longer had spontaneous menstruation at the time of tests, (2) had a history of any cancer treatment for breast cancer (i.e., chemotherapy or endocrine therapy), (3) had a history of another malignancy, (4) had a history of any ovarian surgery, (5) were pregnant, (6) had been diagnosed with any gynecologic problem that might affect $\mathrm{AMH}$ level (i.e., polycystic ovarian syndrome or endometriosis), and (7) had BRCA mutation of undetermined significance. Among the 316 patients included in this study, 264 were $B R C A$-negative and 52 were $B R C A$-positive (27 BRCA1positive and 25 BRCA2-positive). The study was approved by the Institutional Review Board of Samsung Medical Center and exempted from informed consent requirements.

\section{Measurements}

Serum AMH level was measured using AMH ELISA kits (Beckman Coulter, Fullerton, CA, USA) following the manufacturer's directions. The minimum detectable concentration was $0.16 \mathrm{ng} / \mathrm{mL}$, and the inter- and intra-assay coefficients of variation were 5.6 and $5.4 \%$, respectively.

$B R C A$ testing was conducted on peripheral blood using direct sequencing. When pathogenic variants were identified in the genetic tests, all mutations were interpreted utilizing the Human Gene Mutation Database (HGMD; http://www.hgmd. cf.ac.uk/), ClinVar (https://www.ncbi.nlm.nih.gov/clinvar), and Korea ONCOgene Research and Diagnosis (KONCORD; http:// koncord.kr). Mutation nomenclatures from the Breast Cancer Information Core (BIC; http://research.nhgri.nih.gov/bic/) were used for the genetic test reports.

\section{Statistical Analysis}

Statistical analysis was executed using Statistical Analysis System software, version 9.4 (SAS Institute Inc., Cary, NC, USA).

Clinical characteristics and serum AMH level were compared based on the presence of $B R C A$ mutation. Data are presented as median (interquartile range) or number (percentage). Differences between the groups were analyzed using Chi-square test or Fisher's exact tests for categorical variables and Student's $t$-test or Mann-Whitney $U$-test for continuous variables. A $P<0.05$ was considered statistically significant.

Regression analyses were performed to reveal the relationships between serum AMH level and BRCA mutation status after adjusting for age, body mass index, and history of smoking and oral contraceptive use. Linear regression analysis was performed on log-transformed serum AMH levels due to the non-normal distribution of the AMH values. In addition, logistic regression analysis was conducted to examine the association between $B R C A$ mutation status and low AMH level, which represents poor ovarian reserve. For analysis, AMH $<1.2 \mathrm{ng} / \mathrm{mL}$ was considered as poor ovarian reserve based on a previous report (19).

\section{RESULTS}

Table 1 shows the clinical characteristics of the study subjects. The median age was 34 years for both the BRCA-positive and BRCA-negative groups. No differences were found in reproductive or menstrual history, and smoking and alcohol intake did not differ between the two groups. However, the proportions of patients who had progesterone receptor- or human epidermal growth factor receptor 2-positive cancer were significantly higher in the BRCA-positive than in the BRCAnegative group.

Figure 1 shows the median serum AMH level according to $B R C A$-mutation status. Patients with any BRCA mutation had a significantly lower median AMH than those without a mutation ( 2.60 vs. $3.85 \mathrm{ng} / \mathrm{mL}, 32 \%$ decrease, $P=0.004)$. Serum AMH levels of the $B R C A 1(2.56 \mathrm{ng} / \mathrm{mL}, P=0.001)$ and $B R C A 2$ groups (2.64 ng/mL, $P=0.036$ ) were significantly lower than that of $B R C A$-negative group, but no difference was found between the $B R C A 1$ and $B R C A 2$ groups.

Table 2 shows the results of linear regression analysis. Log-transformed AMH was negatively associated with age $(P<0.001)$. After adjusting for age, body mass index, and history of smoking and oral contraceptive use, serum AMH level was still significantly lower in the BRCA-positive group than in the BRCA-negative group $(P=0.043)$. Table 3 shows the results of the logistic regression model evaluating the association between risk of POR and BRCA mutation status. Thirty-five (13.3\%) and 9 (17.3\%) patients had AMH level $<1.2 \mathrm{ng} / \mathrm{mL}$ in the BRCA-negative and BRCA-positive groups, respectively, presenting no statistical difference. After adjusting for age, body mass index, and history of smoking and oral contraceptive use, there was no increased 
TABLE 1 | Clinical characteristics of the study subjects.

\begin{tabular}{lccc}
\hline & $\begin{array}{c}\boldsymbol{B R C A} \text {-negative } \\
(\boldsymbol{n}=\mathbf{2 6 4})\end{array}$ & $\begin{array}{c}\boldsymbol{B R C A} \text {-positive } \\
(\boldsymbol{n}=\mathbf{5 2})\end{array}$ & $\boldsymbol{P}$-value \\
\hline Age, years & $34.0(30.0-36.0)$ & $34.0(30.5-36.0)$ & 0.802 \\
Body mass index, kg/m² & $21.0(19.5-23.0)$ & $20.9(19.3-24.0)$ & 0.820 \\
Age at menarche, years & $14.0(13.0-15.0)$ & $14.0(13.0-15.0)$ & 0.863 \\
Parity & & & 0.635 \\
0 & $148(56.1 \%)$ & $25(48.1 \%)$ & \\
$\geq 1$ & $116(43.9 \%)$ & $27(51.9 \%)$ & \\
Menstruation & & & \\
Regularity & $199(75.4 \%)$ & $45(86.5 \%)$ & 0.064 \\
Duration (days) & $5.0(5.0-6.5)$ & $5.0(4.0-6.5)$ & 0.449 \\
Infertility treatment history & $7(2.7 \%)$ & $1(1.9 \%)$ & 0.760 \\
Receptor status & & & \\
ER (+) & $161(61.0 \%)$ & $27(51.9 \%)$ & 0.224 \\
PR (+) & $133(50.4 \%)$ & $18(34.6 \%)$ & $\mathbf{0 . 0 3 8}$ \\
HER2 (+) & $45(17.1 \%)$ & $0(0.0 \%)$ & $\mathbf{0 . 0 0 1}$ \\
Smoking use & $1(0.38 \%)$ & $0(0.0 \%)$ & 0.657 \\
Alcohol use & $2(0.76 \%)$ & $1(1.9 \%)$ & 0.428 \\
\hline
\end{tabular}

Data are presented as median (IQR) or number (\%). Statistically significant differences are in bold. ER, estrogen receptor. PR, progesterone receptor; HER2, human epidermal growth factor receptor 2.

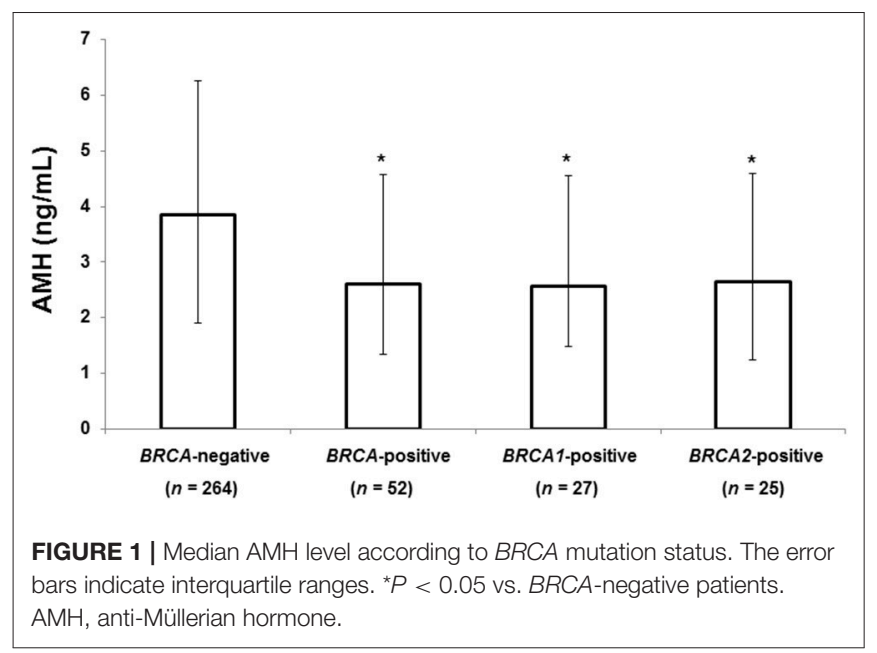

likelihood of POR in the $B R C A$-positive group. In addition, no differences were found between the BRCA1- and BRCA2positive groups in either the linear or logistic regression analysis (data now shown).

\section{DISCUSSION}

This study evaluated the association between BRCA mutation and serum $A M H$ level in breast cancer patients aged $\leq 40$ years. Median AMH was significantly lower in BRCA-positive breast cancer patients compared to $B R C A$-negative patients, but there was no difference in AMH level between the BRCA1-positive and BRCA2-positive groups.
TABLE 2 | Results of linear regression modeling of AMH level.

\begin{tabular}{lccc}
\hline & Parameter estimate & Standard error & $\boldsymbol{P}$-value \\
\hline Intercept & 4.843 & 0.605 & $\mathbf{< 0 . 0 0 1}$ \\
BRCA 1/2 carrier & -0.309 & 0.152 & 0.043 \\
BRCA non-carrier & (ref) & & \\
ADJUSTED VARIABLES & & & \\
Age & -0.092 & 0.014 & $\mathbf{0 . 0 0 1}$ \\
Body mass index & -0.017 & 0.017 & 0.336 \\
Smoking & 0.517 & 1.004 & 0.607 \\
Oral contraceptive use & 0.067 & 0.359 & 0.853 \\
\hline
\end{tabular}

Regression of log transformed AMH level. Statistically significant differences are in bold. $\mathrm{AMH}$, anti-Müllerian hormone.

Our results are similar to those of a previous study demonstrating a trend of lower AMH level (1.8 vs. 2.6 $\mu \mathrm{g} / \mathrm{L}, P=0.109)$ in $29 B R C A$-positive breast cancer patients compared to $72 B R C A$-negative breast cancer patients (17). Since age per se is an important factor determining serum $\mathrm{AMH}$ level, and patients with $B R C A$ mutation show accelerated loss of ovarian follicular reserve and an earlier menopausal age (20), the differences in statistical significance in the studies might have resulted from inclusion of younger patients compared to our study (median age 31 vs. 34 years). Indeed, another study on patients with a median age of 34-36 years reported that $\mathrm{AMH}$ level was significantly lower in $B R C A$-positive breast cancer patients $(1.22$ vs. $2.23 \mathrm{ng} / \mathrm{mL}$; $P<0.001)(16)$.

Several studies have shown that, in non-cancer, healthy subjects, serum AMH level was also significantly lower and ovarian follicles are fewer in BRCA-positive groups than in $B R C A$-negative groups (7-9). However, in one study, $\mathrm{AMH}$ levels were similar between 41 healthy $B R C A$-positive subjects and 324 healthy $B R C A$-negative subjects (12). Overall, the relationship between serum AMH level and $B R C A$ mutation status remains contested.

When we analyzed the BRCA1-positive and BRCA2-positive groups separately in the present study, both had significantly lower AMH level than BRCA-negative patients, but no significant difference was found between the BRCA-positive groups. This finding is in accordance with previous studies presenting no significant difference in serum AMH level between BRCA1positive and $B R C A 2$-positive subjects $(16,17)$. In other studies, however, the serum AMH level was only significantly lower in either the BRCA1-positive (7) or BRCA2-positive (11) group compared to $B R C A$-negative subjects. Further studies are needed to evaluate the associations between each BRCA mutation and ovarian reserve.

In the current study, the prevalence of patients expected to exhibit POR, defined as AMH $<1.2 \mathrm{ng} / \mathrm{mL}$ according to POSEIDON criteria (19), was not different according to BRCA mutation status, and the odds of $\mathrm{AMH}<1.2 \mathrm{ng} / \mathrm{mL}$ did not significantly increase after adjustment for age, body mass index, and history of smoking and oral contraceptive use. The results were the same when POR was assessed using an AMH level 
TABLE 3 | The prevalence of poor ovarian reserve and the results of logistic regression model.

\begin{tabular}{llcc}
\hline & $\begin{array}{l}\text { BRCA-negative } \\
(\boldsymbol{n}=\mathbf{2 6 4 )}\end{array}$ & $\begin{array}{c}\boldsymbol{B R C A} \text {-positive } \\
(\boldsymbol{n}=\mathbf{5 2})\end{array}$ & $\boldsymbol{P}$-value \\
\hline $\begin{array}{l}\text { AMH level, number of } \\
\text { patients (\%) }\end{array}$ & & 0.441 \\
$<1.2 \mathrm{ng} / \mathrm{mL}$ & $35(13.3 \%)$ & $9(17.3 \%)$ & \\
$\geq 1.2 \mathrm{ng} / \mathrm{mL}$ & $229(86.7 \%)$ & $43(82.7 \%)$ & \\
Odds ratio & (reference) & 1.40 & 0.415 \\
$95 \% \mathrm{Cl}$ & (reference) & $0.62-3.17$ & \\
\hline
\end{tabular}

Adjusted for age, body mass index, and history of smoking and oral contraceptive use. $\mathrm{AMH}$, anti-Müllerian hormone; $\mathrm{Cl}$, confidence interval.

of $<1.1 \mathrm{ng} / \mathrm{mL}$ (Bologna criteria) (21) or $<1.0 \mathrm{ng} / \mathrm{mL}$ (17). Although the mean $\mathrm{AMH}$ of $2.64 \mathrm{ng} / \mathrm{mL}$ was lower in $B R C A$ mutation carriers than in non-carriers, the level should be sufficient for pregnancy due to the young age (34 years) of the patients in the current study.

The association between serum AMH level and BRCA mutation may be due to repair of double-strand DNA breaks and maintenance of chromosomal telomeres by BRCA $(4,22,23)$. During reproduction, the telomere is shortened after every cycle of DNA replication, and telomere shortening is related to ovarian aging and reproductive lifespan (24). Furthermore, BRCA1 gene expression decreases significantly with age in human oocytes. In a previous study, BRCA1-mutant mice had fewer oocytes after ovarian stimulation compared to wild-type mice and showed a tendency for DNA damage as a consequence of a deficiency in DNA double-strand break repair (16). Although BRCA2 also repairs DNA double-strand breaks, decreased BRCA2 gene expression typically occurs at the end of the reproductive window, and the proportion of BRCA2 gene expression among all DNA repair genes is small (25).

Our findings have substantial clinical importance in decision-making for young patients with breast cancer and $B R C A$ mutation. From our results, $B R C A$ mutation is an important factor associated with $A M H$ level. Since fertility is attenuated with age, and a risk-reducing salpingooophorectomy should not be delayed over the long-term based on current recommendations, comprehensive, and individualized counseling for fertility preservation, such as oocyte or embryo cryopreservation, should be stressed in this population $(26,27)$.

This study has several strengths. First, the study population was relatively large $(n=316)$, and this is the largest reported

\section{REFERENCES}

1. Ford D, Easton DF. The genetics of breast and ovarian cancer. Br J Cancer. (1995) 72:805-12.

2. Ford D, Easton DF, Peto J. Estimates of the gene frequency of BRCA1 and its contribution to breast and ovarian cancer incidence. Am J Hum Genet. (1995) 57:1457-62. study focusing on breast cancer patients. With the current sample size and difference in serum AMH level, a power of the current study is $98 \%$ with an alpha of 0.05 . Second, our study evaluated the associations between $B R C A$ mutations and decreased ovarian reserve in young breast cancer patients. Associations might differ between those who developed disease and those who were simply mutation-carriers, but most studies have only assessed healthy, non-cancer subjects. In addition, this is the first study of this kind in an Asian population. Genetic background differs across ethnicities; therefore, studies on various ethnicities are clinically important. For example, some studies $(7,12)$ have been performed on patients who carried at least 1 Ashkenazi Jewish founder mutation that is associated with a higher risk of breast and ovarian cancer $(28,29)$, and the results were different from ours. Finally, we analyzed the prevalence of POR according to $B R C A$ mutation.

However, there are some limitations to our study. First, this was a retrospective study performed in one center. Second, we only analyzed serum AMH level to evaluate ovarian reserve. Although serum $\mathrm{AMH}$ level may be a reliable marker for ovarian reserve, addition of antral follicle count or serum follicle-stimulating level would be useful. Third, AMH is generally considered as the best ovarian reserve test, but it does not directly measure the primordial follicle pool. Fourth, although we addressed several factors that could affect serum AMH level, not all the potential confounders affecting serum AMH level were considered for analysis. Moreover, although we measured $\mathrm{AMH}$ and estimated POR, long-term fertility outcomes were not assessed in the present study.

In conclusion, young breast cancer patients with $B R C A$ mutation have significantly lower AMH value, which is indicative of decreased ovarian reserve, compared to BRCAnegative patients. With further studies, our finding can support decision-making for fertility preservation.

\section{ETHICS STATEMENT}

The study was approved by the Institutional Review Board of Samsung Medical Center and exempted from informed consent requirements.

\section{AUTHOR CONTRIBUTIONS}

K-AS, DC, and D-YL were responsible for the concept and design of the study, searching for and analyzing data, and the writing of the manuscript. et al. Average risks of breast and ovarian cancer associated with BRCA1 or BRCA2 mutations detected in case Series unselected for family history: a combined analysis of 22 studies. Am J Hum Genet. (2003) 72:1117-30. doi: $10.1086 / 375033$

4. Venkitaraman AR. Cancer susceptibility and the functions of BRCA1 and BRCA2. Cell. (2002) 108:171-82. doi: 10.1016/S0092-8674(02)00615-3 
5. Broer SL, Broekmans FJ, Laven JS, Fauser BC. Anti-Mullerian hormone: ovarian reserve testing and its potential clinical implications. Hum Reprod Update (2014) 20:688-701. doi: 10.1093/humupd/dmu020

6. Giordano S, Garrett-Mayer E, Mittal N, Smith K, Shulman L, Passaglia C, et al. Association of BRCA1 mutations with impaired ovarian reserve: connection Between Infertility and breast/ovarian cancer risk. J Adolesc Young Adult Oncol. (2016) 5:337-43. doi: 10.1089/jayao.2016.0009

7. Wang ET, Pisarska MD, Bresee C, Chen YD, Lester J, Afshar Y, et al. BRCA1 germline mutations may be associated with reduced ovarian reserve. Fertil Steril. (2014) 102:1723-8. doi: 10.1016/j.fertnstert.2014.08.014

8. Ben-Aharon I, Levi M, Margel D, Yerushalmi R, Rizel S, Perry S, et al. Premature ovarian aging in BRCA carriers: a prototype of systemic precocious aging? Oncotarget. (2018) 9:15931-41. doi: 10.18632/oncotarget.24638

9. Phillips KA, Collins IM, Milne RL, McLachlan SA, Friedlander M, Hickey $\mathrm{M}$, et al. Anti-Müllerian hormone serum concentrations of women with germline BRCA1 or BRCA2 mutations. Hum Reprod. (2016) 31:1126-32. doi: 10.1093/humrep/dew044

10. Oktay K, Kim JY, Barad D, Babayev SN. Association of BRCA1 mutations with occult primary ovarian insufficiency: a possible explanation for the link between infertility and breast/ovarian cancer risks. J Clin Oncol. (2010) 28:240-4. doi: 10.1200/jco.2009.24.2057

11. Johnson L, Sammel MD, Domchek S, Schanne A, Prewitt M, Gracia C. Antimullerian hormone levels are lower in BRCA2 mutation carriers. Fertil Steril. (2017) 107:1256-65.e6. doi: 10.1016/j.fertnstert.2017.03.018

12. Michaelson-Cohen R, Mor P, Srebnik N, Beller U, Levy-Lahad E, Eldar-Geva T. BRCA mutation carriers do not have compromised ovarian reserve. Int $J$ Gynecol Cancer. (2014) 24:233-7. doi: 10.1097/igc.0000000000000058

13. van Tilborg TC, Derks-Smeets IA, Bos AM, Oosterwijk JC, van Golde RJ, de Die-Smulders CE, et al. Serum AMH levels in healthy women from BRCA1/2 mutated families: are they reduced? Hum Reprod. (2016) 31:2651-9. doi: 10.1093/humrep/dew242

14. Grynberg M, Dagher Hayeck B, Papanikolaou EG, Sifer C, Sermondade N, Sonigo C. BRCA1/2 gene mutations do not affect the capacity of oocytes from breast cancer candidates for fertility preservation to mature in vitro. Hum Reprod. (2019) 34:374-9. doi: 10.1093/humrep/dey358

15. Gunnala V, Fields J, Irani M, D'Angelo D, Xu K, Schattman G, et al. BRCA carriers have similar reproductive potential at baseline to noncarriers: comparisons in cancer and cancer-free cohorts undergoing fertility preservation. Fertil Steril. (2019) 111:363-71. doi: 10.1016/j.fertnstert.2018.10.014

16. Titus S, Li F, Stobezki R, Akula K, Unsal E, Jeong K, et al. Impairment of BRCA1-related DNA double-strand break repair leads to ovarian aging in mice and humans. Sci Transl Med. (2013) 5:172ra21. doi: 10.1126/scitranslmed.3004925

17. Lambertini M, Goldrat O, Ferreira AR, Dechene J, Azim HA Jr, Desir J, et al. Reproductive potential and performance of fertility preservation strategies in BRCA-mutated breast cancer patients. Ann Oncol. (2018) 29:237-43. doi: 10.1093/annonc/mdx639

18. Grynberg M, Raad J, Comtet M, Vinolas C, Cedrin-Durnerin I, Sonigo C. Fertility preservation in BRCA-mutated women: when and how? Future Oncol. (2018) 14:483-90. doi: 10.2217/fon-2017-0415
19. Humaidan P, Alviggi C, Fischer R, Esteves SC. The novel POSEIDON stratification of 'Low prognosis patients in Assisted Reproductive Technology' and its proposed marker of successful outcome. F1000Res. (2016) 5:2911. doi: 10.12688/f1000research.10382.1

20. Rzepka-Gorska I, Tarnowski B, Chudecka-Glaz A, Gorski B, Zielinska D, Toloczko-Grabarek A. Premature menopause in patients with BRCA1 gene mutation. Breast Cancer Res Treat. (2006) 100:59-63. doi: 10.1007/s10549-006-9220-1

21. Ferraretti AP, La Marca A, Fauser BC, Tarlatzis B, Nargund G, Gianaroli L. ESHRE consensus on the definition of 'poor response' to ovarian stimulation for in vitro fertilization: the Bologna criteria. Hum Reprod. (2011) 26:1616-24. doi: 10.1093/humrep/der092

22. Lansdorp PM. Repair of telomeric DNA prior to replicative senescence. Mech Ageing Dev. (2000) 118:23-34. doi: 10.1016/S0047-6374(00)00151-2

23. McPherson JP, Hande MP, Poonepalli A, Lemmers B, Zablocki E, Migon E, et al. A role for Brcal in chromosome end maintenance. Hum Mol Genet. (2006) 15:831-8. doi: 10.1093/hmg/ddl002

24. Keefe DL, Marquard K, Liu L. The telomere theory of reproductive senescence in women. Curr Opin Obstet Gynecol. (2006) 18:280-5. doi: 10.1097/01.gco.0000193019.05686.49

25. Oktay K, Turan V, Titus S, Stobezki R, Liu L. BRCA mutations, DNA repair deficiency, and ovarian aging. Biol Reprod. (2015) 93:67. doi: 10.1095/biolreprod.115.132290

26. Paluch-Shimon S, Pagani O, Partridge AH, Abulkhair O, Cardoso MJ, Dent RA, et al. ESO-ESMO 3rd international consensus guidelines for breast cancer in young women (BCY3). Breast. (2017) 35:203-17. doi: 10.1016/j.breast.2017.07.017

27. Peccatori FA, Azim HA Jr, Orecchia R, Hoekstra HJ, Pavlidis N, Kesic V, et al. Cancer, pregnancy and fertility: ESMO clinical practice guidelines for diagnosis, treatment and follow-up. Ann Oncol. (2013) 24(Suppl. 6):vi160-70. doi: 10.1093/annonc/mdt199

28. Levy-Lahad E, Catane R, Eisenberg S, Kaufman B, Hornreich G, Lishinsky E, et al. Founder BRCA1 and BRCA2 mutations in Ashkenazi Jews in Israel: frequency and differential penetrance in ovarian cancer and in breast-ovarian cancer families. Am J Hum Genet. (1997) 60:1059-67.

29. Moslehi R, Chu W, Karlan B, Fishman D, Risch H, Fields A, et al. BRCA1 and BRCA2 mutation analysis of 208 Ashkenazi Jewish women with ovarian cancer. Am J Hum Genet. (2000) 66:1259-72. doi: 10.1086/ 302853

Conflict of Interest Statement: The authors declare that the research was conducted in the absence of any commercial or financial relationships that could be construed as a potential conflict of interest.

Copyright (c) 2019 Son, Lee and Choi. This is an open-access article distributed under the terms of the Creative Commons Attribution License (CC BY). The use, distribution or reproduction in other forums is permitted, provided the original author(s) and the copyright owner(s) are credited and that the original publication in this journal is cited, in accordance with accepted academic practice. No use, distribution or reproduction is permitted which does not comply with these terms. 